\title{
Métodos de diagnóstico y tratamientos utilizados para la ruptura del ligamento cruzado craneal en perros: encuesta a médicos veterinarios de Chile
}

\author{
Diagnostic and treatment methods used for the cranial cruciate ligament rupture in dogs: \\ a survey among veterinarians in Chile
}

\author{
C Fischer", GV Nicolas, AJ Opazo, AF Luzio, IE Troncoso \\ Escuela de Medicina Veterinaria, Universidad Santo Tomás, Sede Concepción, Concepción, Chile.
}

\begin{abstract}
SUMMARY
The cranial cruciate ligament $(\mathrm{CrCL})$ is an important anatomical structure of the stifle that contributes to joint stability. Tears of the CrCL are frequent orthopaedic lesions in dogs and the diagnosis is based on orthopaedic examination. Despite the large number of surgical methods described, no treatment has been universally accepted in veterinary medicine and the treatment is based on the preferences of the surgeon. With regard to diagnosis and treatment, the aim of the present study was to assess the preferred method in Chile to diagnose and treat the rupture of CrCL in dogs. Out of 307 surveys handed out, 206 questionnaires were obtained and $49(23 \%)$ of them stated that one or more methods were performed to diagnose and treat CrCL rupture in dogs, while twenty one $(10,19 \%)$ questionnaires were answered in full. The diagnosis is mainly based on palpation and radiography is the most common complementary method used. Regarding the treatment for the CrCL rupture, the extraarticular surgical technique was the most common method of treatment $(90,47 \%)$. In conclusion, we found out that few veterinarians in Chile diagnose and treat CrCL rupture in dogs. The diagnoses for $\mathrm{CrCL}$ rupture is mostly performed through palpation of the affected stifle while imaging diagnostic methods are scarcely or not applied. The extraarticular technique is the preferred method to treat CrCL rupture in dogs in Chile.
\end{abstract}

Key words: cranial cruciate ligament, dog, survey, diagnosis, treatment.

\section{RESUMEN}

El ligamento cruzado craneal (LCCr) es una estructura anatómica importante en la estabilización de la rodilla, su lesión es considerada una patología ortopédica frecuente en perros. El diagnóstico inicial de la ruptura del LCCr en perros se realiza principalmente a través de la palpación y puede ser complementado con exámenes imagenológicos. A pesar del gran número de métodos quirúrgicos descritos, actualmente en Medicina Veterinaria no hay un método que sea universalmente aceptado, por lo que la elección del tratamiento depende generalmente de las preferencias de cada cirujano. Por esto, el objetivo del presente trabajo fue determinar los métodos de diagnóstico y tratamiento preferentemente utilizados para la ruptura del LCCr por los Médicos Veterinarios. Se realizaron 307 encuestas, obteniéndose 206 respuestas, de las cuales sólo 49 (23\%) profesionales declararon realizar algún tipo de diagnóstico y tratamiento para la ruptura del LCCr en perros. De las respuestas obtenidas, $21(10,19 \%)$ respondieron la encuesta en forma completa. El diagnóstico se basa principalmente en la palpación (100\% y la toma radiográfica $(66,6 \%)$ como método complementario. Dentro de los tratamientos, se destaca mayormente la utilización de la técnica quirúrgica extra-articular (90,47\%), aplicándose en menor grado otros tratamientos. En conclusión, bajo las condiciones de este estudio, son pocos los Médicos Veterinarios que diagnostican y realizan un tratamiento para la ruptura del LCCr en perros. El diagnóstico se basa principalmente en la palpación, siendo los métodos de diagnóstico por imagen poco aplicados o no empleados y el tratamiento de preferencia es quirúrgico, empleándose mayormente la técnica extra-articular.

Palabras clave: ligamento cruzado craneal, perro, encuesta, diagnóstico, tratamiento.

\section{INTRODUCCIÓN}

La ruptura del ligamento cruzado craneal (LCCr) es una de las lesiones ortopédicas más comunes en perros, causante de claudicación del miembro afectado (Johnson y Johnson 1993). La ruptura parcial o total del LCCr produce inestabilidad articular, generando como consecuencia una degeneración progresiva de la articulación y un

Aceptado: 13.06.2013.

* Avda. Prat 897, Concepción, Chile; cfischer@santotomas.cl alto riesgo de lesiones meniscales secundarias (Arnoczky y Marshall 1977).

El diagnóstico de la ruptura del LCCr en perros se puede realizar en base al examen clínico y con la ayuda de herramientas imagenológicas como la ecografía, radiografía, artroscopia, tomografía computarizada y/o resonancia magnética. El diagnóstico clínico detecta principalmente la función anormal del LCCr, siendo la ruptura parcial de difícil diagnóstico (Johnson y Hulse 2002). En la mayoría de los casos, se puede obtener un diagnóstico definitivo con la anamnesis, examen ortopédico y radiografías de la articulación afectada, donde es posible evidenciar alteraciones 
secundarias como efusión articular, o en casos crónicos signos de artrosis, además, nos permite descartar posibles diagnósticos diferenciales (Johnson y Johnson 1993).

En el año 1952, se describe por primera vez un tratamiento quirúrgico para la ruptura del LCCr (Paatsama 1952), y desde ese entonces se han descrito decenas de técnicas con sus respectivas modificaciones (Paatsama 1952, De Angelis y Lau 1970, Slocum y Devine 1984, Smith y Torg 1985, Montavon y col 2002), las que se pueden dividir en tres grandes grupos: técnicas intraarticulares (Paatsama 1952, Arnoczky 1983), extra-articulares (De Angelis y Lau 1970, Smith y Torg 1985) y osteotomías correctivas (Slocum y Slocum 1993, Montavon y col 2002). Hasta la fecha no se ha demostrado la superioridad de una técnica quirúrgica desde el punto de vista clínico (Aragon y Budsberg 2005, Conzemius y col 2005), por lo que la elección de la técnica quirúrgica a utilizar se basa principalmente en las preferencias personales de cada cirujano (Leighton 1999). A pesar del gran desarrollo de nuevas técnicas quirúrgicas e investigaciones, en la actualidad no es posible restaurar la articulación afectada, ni tampoco evitar el desarrollo de una osteoartrosis secundaria (Aragon y Budsberg 2005, Cook 2010). El objetivo de todos los tratamientos para la ruptura del LCCr es reducir la inflamación, el dolor y la inestabilidad articular, con la esperanza de recuperar la función del miembro afectado (Kim y col 2008).

De acuerdo a una encuesta realizada en el año 1999 a cirujanos veterinarios en Estados Unidos, dentro de las técnicas comúnmente utilizadas para el tratamiento de la ruptura del LCCr se encuentran la estabilización con sutura lateral (LSS), la estabilización intra-articular (ICS) (específicamente la técnica denominada "over the top") y la osteotomía correctiva para la nivelación del plato tibial (TPLO) (Leighton 1999).

Debido a que no existe información sobre las preferencias de diagnóstico y tratamiento para la ruptura del ligamento cruzado craneal en perros por los Médicos Veterinarios en Chile, se ha considerado pertinente la actualización de estos datos, siendo el objetivo del presente estudio determinar los métodos de diagnóstico y tratamiento preferentemente utilizados para la ruptura del ligamento cruzado craneal en perros a través de una encuesta realizada a Médicos Veterinarios de Chile.

\section{MATERIAL Y MÉTODOS}

\section{DEFINICIÓN DEL NÚMERO DE ENCUESTADOS}

En una primera instancia se seleccionaron de la guía telefónica nacional disponible en una página $\mathrm{web}^{1}$, todas aquellas Clínicas Veterinarias presentes en esta lista. Al mismo tiempo, se enviaron encuestas a todos los miembros de la Sociedad Chilena de Traumatología y Ortopedia Veterinaria (SOCHITOV). El total de la población a encuestar en el presente estudio fue de 307 profesionales (cuadro 1).

\section{ENCUESTA}

Se desarrolló un tipo de encuesta descriptiva, con un método de muestreo sistemático y no probabilístico. El formato de las respuestas fue cerrado con una escala dicotómica y nominal (Fink 2003). La encuesta se realizó durante los meses de diciembre del año 2012 y enero del año 2013 y constó de preguntas específicas relacionadas con el diagnóstico y tratamiento para la ruptura del LCCr en perros. Las encuestas fueron en base a llamadas telefónicas y a correos electrónicos.

\section{ANÁLISIS ESTADÍSTICO}

Se realizó un análisis estadístico de tipo descriptivo, donde las respuestas de la encuesta sobre los métodos de diagnóstico y tratamientos empleados por los Médicos Veterinarios de Chile fueron analizados y reportados en números enteros con sus respectivos porcentajes.

\section{RESULTADOS Y DISCUSIÓN}

De un total de 307 encuestas realizadas, se obtuvieron $206(67 \%)$ respuestas, de las cuales solo 49 (23\%) declararon realizar algún tipo de diagnóstico y tratamiento para la ruptura del LCCr en perros. De las respuestas obtenidas, $21(10,19 \%)$ respondieron la encuesta en forma completa (cuadro 1).

El porcentaje de respuestas obtenidas (67\%), es alto si se compara con las respuestas obtenidas en otras encuestas realizadas a Médicos Veterinarios en otros estudios y con

Cuadro 1. Número de encuestas realizadas a Médicos Veterinarios de Chile con el número de respuestas y los respectivos porcentajes. Number of surveys carried out among Chilean veterinarians with the number of responses and their respective percentage.

\begin{tabular}{cccccccc}
\hline & $N^{\circ}$ de encuestas & \multicolumn{2}{c}{ Respuestas } & \multicolumn{2}{c}{ Diag/Trat } & \multicolumn{2}{c}{ Encuesta completa } \\
\hline 35 (correo electrónico) & 12 & $34,28 \%$ & 12 & $100 \%$ & 12 & $100 \%$ \\
TOTAL & 272 (teléfono) & 194 & $71,32 \%$ & 37 & $19,07 \%$ & 9 & $24,32 \%$ \\
\hline
\end{tabular}

Diag/Trat: Si realizan algún tipo de diagnóstico y tratamiento para la ruptura del LCCr. 
diferentes tópicos (Hofmeister y col 2008, Heleski y col 2005). La selección de la población tiene relevancia al realizar encuestas no obligatorias, lo que puede influir en los resultados obtenidos en el presente estudio. Al no haber datos respecto al número exacto de Médicos Veterinarios que se dediquen a cirugía de animales menores en Chile, no es posible determinar el grado en el que nuestros datos difieren con la realidad de la población veterinaria en el país.

\section{MÉTODOS DE DIAGNÓSTICO}

En las respuestas relacionadas a los 21 Médicos Veterinarios que respondieron la encuesta completamente, todos (100\%) indican realizar la técnica de palpación a través de la prueba específica de movimiento de cajón como método de diagnóstico para la ruptura del LCCr, información que coincide con lo descrito por Lampman y col (2003) quienes indican que en el $98 \%$ de los casos, se utiliza esta prueba para determinar la lesión, además, 14 $(66,6 \%)$ de ellos realizan como complemento la prueba específica de compresión tibial. Catorce $(66,6 \%)$ de los encuestados, realizan la toma de radiografías como método de diagnóstico complementario para la ruptura del LCCr, porcentaje relacionado con lo descrito por Lampman y col (2003), quienes indican que las radiografías se utilizan en el $45 \%$ de los casos como método de diagnóstico complementario para la ruptura del $\mathrm{LCCr}$ en perros (cuadro 2). Otros métodos complementarios para el diagnóstico de dicha patología como la ecografía y la artroscopia no son de uso común, habiendo sólo $2(9,5 \%)$ y $1(4,7 \%)$ Médicos Veterinarios que declaran utilizar tales métodos de diagnóstico respectivamente, lo cual coincide con lo descrito por Lampman y col (2003), a pesar de que la artroscopia sea un método mínimamente invasivo para diagnosticar y tratar patologías articulares (Johnson y

Cuadro 2. Métodos utilizados para el diagnóstico de la ruptura del ligamento cruzado craneal en perros. Indicando el número de respuestas $\left(\mathrm{N}^{\circ}\right)$ y sus respectivos porcentajes $(\mathrm{n}=21)$.

Diagnostic methods for the cranial cruciate ligament rupture in dogs. number of responses $\left(\mathrm{N}^{\circ}\right)$ and their respective percentage $(\mathrm{n}=21)$.

\begin{tabular}{lcc}
\hline Método de diagnóstico & $\mathrm{N}^{\circ}$ & $\%$ \\
\hline Palpación P.C. & 21 & $(100)$ \\
Palpación C.T. & 14 & $(66,6)$ \\
Radiografía & 14 & $(66,6)$ \\
Ecografía & 2 & $(9,5)$ \\
Artroscopia & 1 & $(4,7)$ \\
Tomografía computarizada & 0 & $(0)$ \\
Resonancia magnética & 0 & $(0)$ \\
\hline
\end{tabular}

P.C: Prueba de cajón, C.T: Compresión tibial.
Hulse 2002). El uso de la tomografía computarizada y de la resonancia magnética para los veterinarios es aún prohibitivo debido a los altos costos de tales procedimientos, lo cual se demuestra en la encuesta, ya que ninguno de los encuestados declara utilizar dichos métodos, a pesar de que en medicina humana la resonancia magnética es actualmente el método de diagnóstico preferido para el diagnóstico de lesiones articulares sinoviales en humanos (Jerram y Walker 2003).

\section{TRATAMIENTOS}

El cuadro 3 resume las respuestas obtenidas en relación a los tratamientos utilizados para la ruptura del LCCr. Del total de 21 personas que respondieron la encuesta, el 100\% declara realizar algún tratamiento quirúrgico para la ruptura del LCCr y 7 de ellos $(33,33 \%)$ también realizan en algunos casos especiales un tratamiento conservador. Vasseur (2003) y Moore y Read (1996) indican que el tratamiento conservador puede considerarse en perros pequeños, al menos para el manejo inicial, lo que contrasta con lo descrito por Hulse (1995), quien recomienda el tratamiento quirúrgico en todos los pacientes por la alta predisposición a sufrir ruptura del LCCr del miembro contralateral, ya que el paciente llevará todo su peso a este miembro para evitar el dolor.

Del total de $21(100 \%)$ encuestas realizadas, cada cirujano indica la utilización de una o más técnicas quirúrgicas favoreciendo principalmente la técnica quirúrgica extra-articular (90,47\%). Leighton (1999) reafirma esta información, indicando que es una técnica útil en pacientes de razas pequeñas y grandes, información que coincide con el estudio realizado por Lampman y col (2003), quien indica que esta técnica se realiza en el $57 \%$ de los pacientes, en comparación con lo descrito por Duerr

Cuadro 3. Métodos utilizados para el tratamiento de la ruptura del ligamento cruzado craneal en perros. Indicando el número de respuestas $\left(\mathrm{N}^{\circ}\right)$ y sus respectivos porcentajes $(\mathrm{n}=21)$.

Methods of repair of the cranial cruciate ligament rupture in dogs. Number of responses $\left(\mathrm{N}^{\circ}\right)$ and their respective percentage $(\mathrm{n}=21)$.

\begin{tabular}{|c|c|c|c|c|}
\hline Tratamiento & & & $\mathrm{N}^{\circ}$ & (\%) \\
\hline Conservador & & & 7 & $(33,33)$ \\
\hline \multirow[t]{6}{*}{ Quirúrgico } & & & 21 & (100) \\
\hline & Intraarticular & & 6 & $(28,57)$ \\
\hline & Extraarticular & & 19 & $(90,47)$ \\
\hline & Osteotomías & & 7 & $(33,33)$ \\
\hline & & TPLO & 4 & (19) \\
\hline & & TTA & 3 & (14) \\
\hline
\end{tabular}

TPLO: Osteotomía niveladora del plató tibial, TTA: Avance de la tuberosidad tibial. 
(2008), quien indica que solo es útil en el $4 \%$ de los pacientes de raza grande que presentan una inclinación elevada del plato tibial. Un estudio más reciente realizado por Cook (2010), quien desarrolla y pone a prueba una nueva técnica extra- articular llamada "Tight- Rope" indica que esta es segura, eficaz y puede ser considerada una opción viable como parte de un plan general de tratamiento para la ruptura de ligamento cruzado craneal.

Las técnicas quirúrgicas a través de las osteotomías correctivas es utilizada por $7(33,33 \%)$ cirujanos veterinarios, cuatro $(19 \%)$ realizan la osteotomía niveladora del plato tibial (TPLO) y 3 (14\%) con el avance de la tuberosidad tibial (TTA), resultado que es similar a lo descrito por Leighton (1999) y Lampman y col (2003) en donde describen el uso de la TPLO en $22,5 \%$ y $22 \%$ respectivamente. Cabe destacar que ninguno de los autores anteriormente nombrados describe la TTA como procedimiento quirúrgico en el grupo de las osteotomías correctivas, lo cual se diferencia del presente trabajo, en donde si hay cirujanos que declaran utilizar la TTA como procedimiento. Dicha diferencia se debe principalmente a que la TTA es una técnica descrita recién el año 2002 por Montavon, por lo que el uso de dicho procedimiento no se realizaba el año en donde Leighton (1999) hizo su investigación y era poco común cuando Lampman y col (2003) escribió su artículo.

Finalmente, la técnica intraarticular es utilizada por $6(28,57 \%)$ cirujanos, lo cual también se asemeja a lo descrito en su momento por Leighton (1999), en donde $31,5 \%$ de los encuestados utiliza algún método intraarticular para el tratamiento de la ruptura del LCCr en perros.

De acuerdo a los resultados obtenidos en la encuesta realizada, se puede concluir que bajo las condiciones de este estudio solo un bajo porcentaje de Médicos Veterinarios realiza un diagnóstico y tratamiento para la ruptura de LCCr en perros. El diagnóstico de la ruptura del LCCr en perros se basa principalmente en la utilización de la palpación, a través de la prueba específica de movimiento de cajón y la toma radiográfica como método de diagnóstico complementario, no haciendo uso o muy bajo de otros métodos diagnósticos como la artroscopia, ecografía y resonancia magnética. Dentro de los tratamientos utilizados, se destaca mayormente la utilización de la técnica quirúrgica extra- articular.

\section{REFERENCIAS}

Aragon C, S Budsberg. 2005. Applications of evidence-based medicine: cranial cruciate ligament injury repair in the dog. Vet Surg 34, 93-98.

Arnoczky S, J Marshall. 1977. The cruciate ligaments of the canine stifle: an anatomical and functional analysis. Am J Vet Res 38, 1807-1814.

Arnoczky S. 1983. Cranial cruciate ligament repair. In: Bojrab MJ (ed). Current techniques in small animal surgery. 2 ed. Lea \& Febiger, Philadelphia, USA, Pp 647-650.

Conzemius M, R Evans, MF Besancon, C Horstman, WD Ho- efle, MA Nieves, SD Wagner. 2005. Effect of surgical technique on limb function after surgery for rupture of the cranial cruciate ligament in dogs. J Am Vet Med Assoc 226, 232-236.

Cook JL. 2010. Clinical Comparison of a novel extracapsular stabilization procedure and tibial plateau leveling osteotomy for treatment of cranial cruciate ligament deficiency in dogs. Vet Surg 39, 315-323.

De Angelis M, R Lau. 1970. A Lateral retinacular imbrications technique for the surgical correction of anterior cruciate ligament rupture in the dog. J Am Anim Hosp Assoc 157, 79-84.

Duerr F, C Duncan, R Savicky, R Park, E Egger, R Palmer. 2008. Comparison of surgical treatment options for cranial cruciate ligament disease in large-breed dogs with excessive tibial plateau angle. Vet Surg 37, 49-62.

Fink A. 2003. The survey kit. $2^{\text {nd }}$ ed. Sage Publications, California, USA.

Heleski CR, AG Mertig, AJ Zanella. 2005. Results of a national survey of US veterinary college faculty regarding attitudes toward farm animal welfare. J Am Vet Med Assoc 226, 1538-1546.

Hofmeister EH, BF Thompson, BM Brainard, S Kegge, S Kube, CM Egger, C Jehn, B Green. 2008. Survey of academic veterinarians'clinical practice in cardiopulmonary cerebral resuscitation. J Vet Emerg Crit Care 18, 142-152.

Hulse D. 1995. The stifle joint. In: Olmstead ML (ed). Small animal orthopedics. Mosby-Year Book Inc. Missouri, USA, Pp 404-411.

Jerram R, A Walker. 2003. Cranial cruciate ligament injury in the dog: pathophysiology, diagnosis and treatment. $N Z$ Vet J 51, 149-158.

Johnson A, D Hulse. 2002. Cranial cruciate ligament rupture. In: Fossum TW (ed). Small Animal Surgery, $2^{\text {nd }}$ ed. Mosby-Year Book Inc., Missouri, USA, Pp 1110-1122.

Johnson JM, AL Johnson. 1993. Cranial cruciate ligament rupture. pathogenesis, diagnosis, and postoperative rehabilitation. Vet Clin North Am Small Anim Pract 23, 717-733.

Kim S, A Pozzi, M Kowaleski, D Lewis. 2008. Tibial osteotomy for cranial cruciate ligament insufficiency in dogs. Vet Surg 37, 111-125.

Lampman T, E Lund, A Lipowitz. 2003. Cranial cruciate disease: current status of diagnosis, surgery, and risk for disease. Vet Comp Orthop Traumatol 16, 122-126.

Leighton RL. 1999. Preferred method of repair of cranial cruciate ligament rupture in dogs: a survey of ACVS diplomates specializing in canine orthopedics. Am Coll Vet Surg 28, 194.

Montavon P, D Damur, S Tepic. 2002. Advancement of the tibial tuberosity for the treatment of cranial cruciate deficient canine stifle. Proceedings of the 1st World Orthopedic Veterinary Congress, Munich, Germany, Pp 2152.

Moore K, R Read. 1996. Rupture of the cranial cruciate ligament in dogs. Part II. Diagnosis and management. Compendium of continuing education for the practicing veterinarian 18, 223-233.

Paatsama S. 1952. Ligament injuries in the canine stifle joint. A clinical and experimental study. PhD Thesis, University of Helsinki, Helsinki, Finland.

Slocum B, T Devine. 1984. Cranial tibial wedge osteotomy: A technique for eliminating cranial tibial thrust in cranial 
cruciate repair. J Am Vet Med Assoc 184, 564-569.

Slocum B, T Slocum. 1993. Tibial plateau leveling osteotomy for repair of cranial cruciate ligament rupture in the canine. Vet Clinic North Am Small Anim Pract 23, 777-795.

Smith G, J Torg. 1985. Fibula head transposition for repair of the cruciate-deficient stifle in the dog. J Am Vet Med Assoc 187, 375-383.

Vasseur P. 2003. Stifle joint. In: Slatter D (ed). Textbook of Small Animal Surgery. $3^{\text {rd }}$ ed. Saunders. Philadelphia, USA, Pp 2095-2116. 
